RELATO DE CASO

ISSN 1677-5090

(C) 2019 Revista de Ciências Médicas e Biológicas

http://dx.doi.org/10.9771/cmbio.v18i3.34186

\title{
Adesão à terapia fonoaudiológica por paciente com disfagia orofaríngea: relato de caso
}

\author{
Adherence to speech therapy by a patient with oropharyngeal dysphagia: case report
}

\author{
Anderson Ferreira dos Santos ${ }^{1}$, Carla Steinberg ${ }^{2}$, Ana Caline Nóbrega da Costa ${ }^{3 *}$ \\ Graduado em Fonoaudiologia pela Universidade do Estado da Bahia (UNEB). Mestrando do Programa de Pós- \\ Graduação em Processos Interativos de Órgãos e Sistemas do Instituto de Ciências da Saúde, da Universidade \\ Federal da Bahia (UFBA); ${ }^{2}$ Doutora em Processos Interativos dos órgãos e Sistemas, pela UFBA. Mestre em \\ Fonoaudiologia, pela Pontifícia Universidade Católica de São Paulo (PUC/SP). Docente do curso de Bacharelado em \\ Fonoaudiologia, da UFBA; ${ }^{3}$ Doutora e Mestre em Medicina e Saúde, pela UFBA. Docente do curso de Bacharelado \\ em Fonoaudiologia, da UFBA.
}

\begin{abstract}
Resumo
Introdução: os sujeitos com disfagias orofaríngeas podem apresentar diversos riscos, entre eles os riscos respiratórios. O diagnóstico preciso e o tratamento adequado apresenta resultados satisfatórios para a segurança do paciente, evitam impactos negativos sobre a sua saúde e qualidade de vida e, para isso, a adesão a terapia é fundamental. Objetivos: investigar a adesão ao atendimento fonoaudiológico de uma paciente com disfagia, caracterizar a adesão ao tratamento e pesquisar os motivos que interferem na adesão e não adesão ao tratamento fonoaudiológico. Metodologia: trata-se de um estudo de caso desenvolvido no ambulatório de Fonoaudiologia, do pavilhão de ambulatórios Professor Francisco Magalhães Neto, do Complexo Hospitalar Universitário Professor Edgar Santos (HUPES). O formato adotado é de relato de caso, seguido de discussões à luz de contribuições clínicas e outras publicações. Resultados: o caso apresentado se refere a dificuldade de adesão a terapia por conta de questões socioeconômicas. Conclusão: apesar da importância do atendimento fonoaudiológico, a paciente e seus familiares encontram barreiras sociais para sua adesão integral, o que acarreta em uma assistência incompleta à demanda apresentada pela doença de base.

Palavras-chave: Disfagia. Terapia fonoaudiológica. Adesão terapêutica.
\end{abstract}

\begin{abstract}
Introduction: oropharyngeal dysphagia presents several risks and respiratory complications for affected patients. Accurate diagnosis and appropriate treatment provide satisfactory results for patient safety, avoid negative impacts on their health and quality of life and for this, adherence to therapy is essential. Objectives: to investigate the adherence of dysphagia patients to speech therapy, to characterize treatment adherence and search the reasons that interfere with adherence and non-adherence to speech therapy. Methodology: This is a cross-sectional, observational and exploratory study, developed at the Speech-Language Pathology Outpatient Clinic of the Professor Francisco Magalhães Neto Outpatient Clinic of the Professor Edgar Santos University Hospital Complex (HUPES). The format adopted is a case report, followed by discussions in the light of clinical contributions and other publications. Results: the case presented here is a relative difficulty in adhering to therapy due to socioeconomic issues. Conclusion: Despite the importance of speech therapy, patients and their families find social barriers to their full adherence, which leads to incomplete assistance to the demand presented by the underlying disease.
\end{abstract}

Keywords: Dysphagia. Speech therapy. TherapeuticAdherence

\section{INTRODUÇÃO}

As disfagias orofaríngeas podem ocorrer por alterações mecânicas, neurogênicas e/ou psicogênicas, por conta de mudanças na fase oral ou faríngea da deglutição (FERNANDES; ODA, 2017).

Dentre os recursos disponíveis para tratamento e acompanhamento das disfagias, estão ações cirúrgicas, medicamentosas, indicação de vias alternativas de alimentação e terapia de habilitação/reabilitação da deglutição,

Correspondente/ Corresponding: Ana Caline Nóbrega da Costa - End: Instituto de Ciências da Saúde - ICS. Avenida Reitor Miguel Calmon, s/n, Canela, Salvador - BA. CEP: 40231-300 - Tels: (71) 99975-9905 —E-mails: anacalinen@gmail.com

Rev. Ciênc. Méd. Biol., Salvador, v. 18, n. 3, p. 411-415, set./dez. 2019 que envolve também ajuste de consistência dos alimentos (FURKIM; SACCO, 2008).

O tratamento requer uma avaliação adequada para um diagnóstico preciso, o que leva a resultados mais satisfatórios, no tocante à eficiência e à eficácia do tratamento para a segurança ao paciente, visto que a disfagia acarreta desnutrição, desidratação, riscos de complicações respiratórias e até quadros de depressão, a depender dos pacientes e das suas variáveis sociais (PADOVANI et al., 2007).

A clínica fonoaudiológica para a disfagia está dividida em dois ramos de atendimento, a reabilitação e o gerenciamento. Ambos objetivam, dentre outros aspectos, a reintrodução da alimentação e hidratação por via oral, 
se possível, com segurança, de modo a ofertar o suporte nutricional adequado, sem riscos de penetração e/ou aspiração laringotraqueal e sem os riscos associados de morbidade e mortalidade, além da redução de riscos visando a manutenção da dieta por via oral pelo maior tempo possível e/ou até a indicação, no momento mais oportuno, da via alternativa/suplementar (TURRA, 2014).

O cotidiano da clínica de reabilitação em disfagia apresenta casos de abandono e baixa adesão à terapia, com considerável número de desajustes entre o orientado pelo profissional e o seguido/cumprido pelos pacientes, pais e/ou cuidadores. Vale ressaltar que a baixa adesão ao tratamento pode prejudicar a evolução clínica do paciente e acarretar em consequências emocionais, sociais, e econômicas, entretanto, a adesão à terapia pelos pacientes com disfagia ainda é pouco descrita.

Para a Organização Mundial de Saúde (OMS) a adesão ao tratamento é um conjunto relacionado ao grau de concordância entre o comportamento de uma pessoa e as orientações do médico ou de outro profissional de saúde; é um fenômeno onde interagem fatores referentes ao paciente, à doença, ao tratamento, socioeconômicos e à equipe de saúde (WHO, 2003).

A adesão a um tratamento pode ser resumida como uma etapa para se alcançar um objetivo, manter ou melhorar a saúde, visando reduzir os sinais e sintomas de uma doença (PARO; VIANNA; LIMA, 2013), por conseguinte, pode-se também inferir, que a baixa adesão pode ter relação com as peculiaridades do tratamento, com a própria doença, com o paciente e até com a relação entre o profissional da saúde e o paciente (KLEIN; GONÇALVES, 2005). Assim, pode-se propor o seguinte conceito de adesão:

\section{A adesão não se reduz a um ato de volição pessoal. É um processo intimamente asso- ciado à vida, que depende de uma série de intermediações que envolvem o cotidiano da pessoa, a organização dos processos de trabalho em saúde e a acessibilidade em sentido amplo que inclui os processos que levam - ou não - ao desenvolvimento da vida com dignidade (BERTOLOZZI et al., 2009).}

Na clínica fonoaudiológica fala-se muito sobre adesão e não adesão dos pacientes ao tratamento, entretanto, pouco se encontra na literatura estudos a respeito dessa informação, caracterizando dados precisos e vivenciais dos pacientes. De fato, há muita investigação relacionada às doenças crônicas, entretanto, a adesão à terapia fonoaudiológica, no tocante ao tratamento das disfagias, sintoma que agrava o quadro clínico e pode aumentar a letalidade de sujeitos com doenças neuro degenerativas, é pouco explorada.

Assim sendo, este estudo objetiva investigar a adesão ao atendimento fonoaudiológico de uma paciente com disfagia, caracterizar a adesão ao tratamento e pesquisar os motivos que interferem na adesão e não adesão ao tratamento fonoaudiológico.

\section{METODOLOGIA}

Trata-se de um estudo de caso, desenvolvido no ambulatório de Fonoaudiologia do pavilhão de ambulatórios Professor Francisco Magalhães Neto, do Complexo Hospitalar Universitário Professor Edgar Santos (HUPES), utilizando um roteiro de entrevista semiestruturada com 31 questões, respondida pela paciente e pela acompanhante, abordando aspectos de identificação, socioeconômicos, diagnósticos, familiares, relações com o processo terapêutico, alimentação e percepção sobre qualidade de vida. Foi aplicada também a escala de depressão geriátrica - GDS (YESAVAGE et al., 1983), que rastreia através de 15 perguntas, a depressão no idoso.

Após leitura e explicações, a familiar assinou o Termo de Consentimento Livre e Esclarecido (TCLE) com o consentimento da sua genitora. Todo o depoimento/entrevista foi registrado em arquivo de áudio, no formato $\mathrm{mp} 3$ e depois transcrito em arquivo de texto para uma análise qualitativa, na qual o foco foram os relatos relacionados à adesão terapêutica.

$O$ estudo foi realizado de acordo com as normas estipuladas na resolução $n$ ㅇ 466/12, de 12 de dezembro de 2012 da Comissão Nacional de Saúde e aprovado pelo Comitê de Ética em Pesquisa do Complexo Hospitalar Professor Edgar Santos sob registro CAAE: 19483119.8.0000.0049.

\section{RELATO DE CASO}

H.E.S., 78 anos, mulher negra, alfabetizada, oriunda do interior da Bahia, moradora de uma comunidade periférica de Salvador, viúva, aposentada, mãe de uma filha e um filho, ambos adultos. Tem diagnóstico de doença de Parkinson (DP) desde 2017, em acompanhamento fonoaudiológico desde o segundo semestre de 2018, encaminhada pelo médico neurologista; tem conhecimento do seu diagnóstico, mas relata não saber nada sobre a doença. Acompanhada pela filha, S.S.S., 55 anos, mulher negra, doméstica, alfabetizada, nascida na cidade de Salvador, moradora de uma comunidade periférica de Salvador, casada, mãe de um filho, que ajuda a cuidar da avó.

A referida paciente é acompanhada atualmente pelas especialidades de Fonoaudiologia, Neurologia, Cardiologia e Pneumologia do Complexo HUPES.

A acompanhante relata que foi informada que a mãe tem indicação de atendimento por no mínimo duas vezes na semana e assim iniciou o acompanhamento fonoaudiológico, porém por pouco tempo. S.S.S., é quem cuida da paciente e moram na mesma casa atualmente, a mesma é empregada doméstica e começou a enfrentar problemas no trabalho porque precisava sair mais cedo nos dois dias de atendimento, passando a não ser liberada 
pelos seus patrões e com "ameaça de perder o emprego". Para solucionar o problema no trabalho e não parar os atendimentos, o caminho encontrado pela filha foi que esse atendimento acontecesse quinzenalmente, ou seja, duas vezes ao mês. Relata não entender bem a doença, como ela iniciou, suas causas e não buscou informações sobre. No tocante à alimentação, a paciente mantém dieta por via oral em múltiplas consistências, relatando dificuldade para engolir, mastigação lenta, deglutição fraca, com engasgos, mas sem relato de pneumonia reforçados por S.S.S.

Tem uma profissional da Fisioterapia que a ajuda na realização dos exercícios passados pela terapeuta da Fonoaudiologia como um aporte na manutenção dos exercícios.

Quando questionada sobre a frequência no atendimento, a filha diz: "é porque eles não entendem que eu tenho que trazer minha mãe, [...] eles aceitam que eu saia de 15 em 15, mesmo assim ainda falando".

Sobre a justificativa dos patrões ela desabafa:

Eles dizem que estou saindo demais para levar minha mãe para o médico, é porque para eles não existe isso né, de levar a mãe para o médico. Me perguntam por que não tenho uma pessoa, aí eu sempre digo que eu não tenho condições de pagar a uma pessoa para ficar trazendo ela para o médico, porque se eu for fazer isso, e aí como é que fica as coisas para trazer ela, porque ela não anda de ônibus, [...] então é uma dificuldade assim, porque tem que comprar remédio para ela, tudo isso, tem as coisas dela. E aí, se eu for pagar a uma pessoa como vou fazer isso? É complicado! Mas tô conseguindo, graças a Deus, orando, pedindo a Deus e muito né para ele me ajudar, porque eu também não quero ficar desempregada, mas puder trazer ela para acompanhar ela, porque eu sei a dificuldade que ela passava na hora de comer, que ela engasgava, que ela chegava a chorar, se urinar também quando engasga, então tudo isso eu via minha mãe passar. Então quando eu consegui eu fiquei muito feliz, então para poder interromper o tratamento dela por causa do trabalho fica difícil.

A fala de S.S.S., a filha da paciente, é carregada de emoções, medos e ao mesmo tempo de certa revolta por não poder fazer pela sua mãe tudo que deseja. A sua expressão facial e corporal, no momento da entrevista, pode confirmar essas suposições.

No que tange à adesão as orientações/recomendações feitas, H.E.S e S.S.S., relatam que seguem todas as orientações sobre a alimentação e os exercícios, tendo mais dificuldade de realizar os exercícios na frequência indicada.
Após a entrevista, a paciente diz que está chateada, porque não consegue mais sair e andar como fazia, mas diz-se feliz. Respondeu a todas as perguntas da entrevista, entretanto se posiciona com reserva e em falas curtas quando the é dada a palavra para fala espontânea. $\mathrm{Na}$ Escala de Depressão Geriátrica (GDS) ela obteve 5 (cinco) pontos, o limite máximo para não suspeição de depressão, respondendo afirmativamente as questões 2,3,4,9 e 15 .

\section{DISCUSSÃO}

Neste relato de caso, de uma paciente com DP, de 78 anos, observamos que a adesão ao tratamento fonoaudiológico não foi integral. Três aspectos proporcionam peculiaridades e especificidades no entendimento da adesão, na tentativa de identificar potencias para a consecução do tratamento: a concepção de saúde-doença apresentada pela pessoa que apresenta a enfermidade; o lugar social ocupado pela pessoa doente; o processo de produção da saúde (BERTOLOZZl et al., 2009). Nesse sentido, não identificamos no seu relato o quanto os referidos aspectos interferiram na sua adesão, entretanto observa-se que o aspecto socioeconômico tem grande impacto nesse processo. Além disso, notamos que no aspecto alimentação, ajuste de consistências e dieta oral de modo geral, H.E.S., e S.S.S., seguem integralmente e não relatam queixas sobre esse ponto. Sobre os exercícios propostos, H.E.S. relata que as vezes esquece, mas que faz todos os dias, mesmo não sendo cobrada. Diz que gosta dos exercícios e os demonstra durante a entrevista, sendo realizados corretamente.

A doença de Parkinson é definida como distúrbio neurológico progressivo, caracterizado principalmente pela degeneração das células (neurônios) da camada ventral da parte compacta da substância negra e do lócus ceruleus. Tal degeneração resulta na diminuição da produção de dopamina, produzindo um conjunto de sintomas caracterizados principalmente por distúrbios motores (GONÇALVES; ALVAREZ; ARRUDA, 2007).

Doenças crônicas e progressivas caracterizam-se pela manutenção dos sintomas, desenvolvendo efeitos progressivamente, que podem provocar sofrimento e incômodos nos pacientes e nos familiares, afetando aspectos físicos que acarretam fatores emocionais, por conta das limitações, agregada a falta de assistência preventiva e de promoção da saúde (PETERNELLA; MARCON, 2009), onde são resgatadas ou ativadas as redes de apoio familiar que esse relato apresenta, associando o apoio dos familiares, como a filha e neto da paciente.

As redes de apoio familiar, de relações sociais, permeiam por toda a vida dos indivíduos e são transformadas ao longo do ciclo vital. Tais relações são as possibilidades de apoio em momentos de crise (JULIANO; YUNES, 2014). Ao enfrentar uma nova etapa, a família identifica e aciona sua rede de apoio de forma variável para esse enfrentamento, o que pode gerar tensões e desconfortos no indivíduo e na família como forma de reorganizar e buscar 
um enfrentamento saudável para dar conta das demandas presentes (SEIBEL et al., 2017). No caso de H.E.S, a filha S.S.S., buscou, dentre outras formas, enfrentar as tensões geradas pela DP em sua mãe com aproximação, quando decide que elas passariam a viver na mesma residência, o que para a H.E.S., foi encarado com naturalidade e zelo por parte da filha. Além disso, S.S.S., ampliou a rede de apoio familiar envolvendo seu filho, neto de H.E.S., que mesmo morando em outra residência, em outro bairro, aderiu, dentro das suas possibilidades, à rede em prol da melhora e da qualidade de vida para sua avó.

A história de H.E.S., apesar de pouco contada nos meios acadêmicos é muito comum na clínica fonoaudiológica e fácil de ser encontrada em ambientes de terapia de reabilitação. Além dos determinantes sociais envolvidos tanto na vida da paciente quanto da filha, em sua família de modo geral, destacou-se o contexto social das relações de trabalho e seu envolvimento com a rede de apoio que foi acionada. Ter indicação de atendimento e não cumpri-lo é, no mínimo, aumentar os riscos de morbimortalidade por conta das consequências que a doença acarreta e essa é a realidade que tenta ser contornada pela cuidadora.

Do ponto de vista fonoaudiológico, a respiração, a fala e a deglutição de H.E.S., carecem de atenção, visto que ela corre riscos respiratórios que podem ser prevenidos e/ou retardados com a terapia, mantendo o prazer de alimentação por via oral, o que Ihe daria melhor qualidade de vida e até mesmo, poder ressignificar sua condição, restaurando o entusiasmo que foi abalado com a limitação de não fazer tudo que fazia antes.

Durante toda a entrevista observamos o cuidado e a atenção de S.S.S., para a fala de sua genitora, ao tempo que sua expressão, também observada com atenção pela sua mãe, era emotiva, por vezes com voz "trêmula", oscilando entre exaltação e contenção, denotando medo e "incômodo" pelas limitações que encontra para ajudar sua mãe. Entretanto, mesmo sabendo das suas limitações, já que ela é o membro acionado da rede de apoio, está disposta a enfrentar, dentro dos seus limites, o que for preciso para manter sua mãe bem.

A família ao se envolver no caso, vivencia todas as mudanças e sentimentos gerados pelo diagnóstico de uma doença crônica no seu meio. Na DP, as dificuldades são intensificadas, porque além da cronicidade ela é uma doença neurodegenerativa e progressiva, afetando os aspectos físico, mental e social do individuo, acarretando em dependências e necessidades vivenciadas também pela família. Variados sentimentos se apresentam, tanto na família como no paciente, praticamente somente os negativos como a não aceitação, tristeza, revolta, raiva, além de, por parte do paciente, ser reforçado o medo da dependência, a depressão e decepção quando se há o entendimento do prognóstico incurável da doença (PETERNELLA; MARCON, 2009).

Vale ressaltar ainda, que H.E.S., na avaliação da Escala de Depressão Geriátrica, está próxima a entrar na faixa de suspeita de depressão. Suas respostas, pontuaram na em questões que denotam um caminho de reclusão, o que chama atenção da família para esse sentimento, ao mesmo tempo que gera receios sobre como a paciente pode encarar o seu estado à medida que a doença for avançando.

\section{CONCLUSÃO}

O caso relatado apresentou uma história forte e constante na clínica fonoaudiológica, em especial nos ambientes de saúde do serviço público, apesar de pouco relatada pela comunidade acadêmica. A paciente cursa com o quadro característico da doença e tem a adesão ao tratamento prejudicada por fatores econômicos e sociais. Estratégias tem sido usadas e orientadas elas profissionais responsáveis pelo acompanhamento, de modo a reduzir os impactos da não adesão à terapia fonoaudiológica, por falta da frequência ideal de atendimentos, explicada pelo relato da cuidadora.

Desta forma, o presente relato reforça a necessidade da discussão sobre a adesão ou não adesão à fonoterapia, visando identificar os motivos para os mesmos e pensar o que pode ser feito para se adaptar à realidade dos pacientes e os determinantes sociais aos quais estão envolvidos. Como terapeutas e voltados à humanização dos atendimentos, é importante a manutenção da escuta sensível, para pontuar caso a caso, e pensar individualmente caminhos que possam atender às demandas em sua maior integralidade.

\section{REFERENCIAS}

BERTOLOZZI, M. R. et al. Os conceitos de vulnerabilidade e adesão na Saúde Coletiva. Rev. Esc. Enferm. USP, São Paulo, v. 43, p.1326-30, 2009.

FERNANDES, A. M. F.; ODA, A. L. Disfagia Neurogênica. DEDIVITIS, R. A.; SANTORO, P. P.; ARAKAWA-SUGUENO, L. Manual prático de disfagia: diagnóstico e tratamento. Rio de Janeiro: Revinter, 2017.

FURKIM, A.M.; SACCO, A. B. de F. Eficácia da fonoterapia em disfagia neurogênica usando a escala funcional de ingestão por via oral (FOIS) como marcador. Rev. CEFAC, São Paulo, v. 10, n. 4, p. 503-512, 2008. Disponível em: https://www.redalyc.org/pdf/1693/169317451010.pdf. Acesso em: 11 out. 2019.

GONÇALVES, L. H. T.; ALVAREZ, A. M.; ARRUDA, M. C. Pacientes portadores da doença de Parkinson: significado de suas vivências. Acta paul. enferm., São Paulo, v. 20, n.1, p. 62-68, 2007. Disponível em: http://www. scielo.br/pdf/\%0D/ape/v20n1/a11v20n1.pdf. Acesso em: 11 out. 2019.

JULIANO, M. C. C.; YUNES, M. A. M. Reflexões sobre rede de apoio social como mecanismo de proteção e promoção de resiliência. Ambiente \& Sociedade, [s.I], v. 17, n. 3, p. 135-154, 2014.

KLEIN, J. M.; GONÇALVES, A. da G. A. A adesão terapêutica em contexto de cuidados de saúde primários. Psico USF, Bragança Paulista, v. 10, n. 2, p.113-20, 2005. Disponível em: https://www.redalyc.org/ pdf/4010/401036064002.pdf. Acesso em: 11 out. 2019.

PADOVANI, A. R. et al. Protocolo fonoaudiológico de avaliação do risco para disfagia (PARD). Rev. Soc. Bras. Fonoaudiol., São Paulo, v.12, n. 3, p. 199-205, 2007.

PARO, C.A.; VIANNA, N.G.; LIMA, M.C.M.P. Investigando a adesão ao atendimento fonoaudiológico no contexto da atenção básica. Rev. 
CEFAC, São Paulo, v. 15, n.5, p. 1316-1324, set./out. 2013.

PETERNELLA, F. M. N.; MARCON, S. S. Descobrindo a Doença de Parkinson: impacto para o parkinsoniano e seu familiar. Rev. bras. enferm., Brasília, v. 62, n. 1, p. 25-31, 2009. Disponível em: https://www.redalyc. org/pdf/2670/267019601005.pdf. Acesso em: 11 out. 2019.

SEIBEL, B. L. et al. Rede de apoio social e funcionamento familiar: estudo longitudinal sobre famílias em vulnerabilidade social. Pensando fam., Porto Alegre, v. 21, n. 1, p. 120-136, 2017. Disponível em: https:// www.lume.ufrgs.br/bitstream/handle/10183/185176/001081842. pdf?sequence=1\&isAllowed=y. Acesso em: 11 out. 2019.

TURRA, G. S. Intervenção fonoaudiológica em pacientes com disfagia, pós intubados e sem morbidades neurológicas. 2014. Tese (Doutorado) - Universidade Federal do Rio Grande do Sul, Faculdade de Medicina, Programa de Pós-Graduação em Medicina: Ciências Médicas, Porto Alegre, 2014. Disponível em: https://www.lume.ufrgs.br/bitstream/ handle/10183/97953/000919776. pdf? sequence=1\&isAllowed=y. Acesso em: 11 out. 2019.

WORLD HEALTH ORGANIZATION (WHO). Adherence to long-termtherapies: evidence for action. Geneva: WHO, 2003.

YESAVAGE, J. A. et al. Development and validation of a geriatric depression screening scale: a preliminary report. J. psychiatr. res., Oxford, v. 17, n. 1, p. 37-49, 1983. Disponível em: https://www.sciencedirect.com/ science/article/abs/pii/0022395682900334. Acesso em: 11 out. 2019.

Submetido em: 04/11/2019

Aceito em: 29/11/2019 